\title{
Auxiliary field in conformal gauge theory
}

\author{
James T. Wheeler \\ Department of Physics, Utah State University, Logan, Utah 84322
}

(Received 1 May 1991)

\begin{abstract}
When the full conformal algebra is gauged there arises a gauge field for inverse translations in addition to the usual translational gauge field. By considering every scale-invariant action constructible from the curvatures of the conformal group and the metric, we show that when the gauge field of the usual translations is identified as the vierbein, the gauge field of inverse translations may always be eliminated by its own field equation. After this elimination, every torsion-free, scale-invariant action reduces to a linear combination of the square of the conformal curvature tensor $C_{\alpha \beta \mu \nu}$ and the square of the Weyl field strength $C_{\alpha \beta}$.
\end{abstract}

Global scale invariance is an everyday part of science. Every time we change from meters to centimeters or set Planck's constant equal to one, we make use of our ability to set the scale of spacetime measurements arbitrarily. Equally clear, but less frequently used, is our ability to choose this scale locally, assigning a different length standard at each point of spacetime.

The gauge field of local scale change is called the Weyl vector. When its field strength is nonvanishing, physical objects can experience a measurable length change, and the spacetime geometry becomes non-Riemannian. In the resulting Weyl geometry [1], there may be no extremal paths, since lengths may be arbitrarily rescaled.

Recently, this absence of preferred paths has been turned to advantage by using Weyl geometry as a model of the motion of a charged quantum-mechanical particle [2]. Since no extremal path exists, it is assumed that the particle "diffuses" preferentially along those paths for which its physical magnitude changes the least. Meaningful physical predictions arise only when the particle field is multiplied by its Weyl conjugate to produce a scale-invariant number. This accounts for the quadratic character of the probability density in quantum mechanics. In the special case when the Weyl field does allow preferred motion, that motion is governed by the Lorentz force law.

To be convincing, the scope of any such model must be broadened to include many-particle systems and the remaining two fundamental forces. The natural first step in this generalization is to extend the symmetry group to the conformal group and look in detail at its gauge theory. It is well known that the conformal group is the maximal group of transformations which leaves relative lengths invariant, while allowing scale changes.

In the present work, we will be overtly concerned with establishing the form of the action in conformal gauge theory. Indeed, we succeed in extending to all possible scale-invariant actions some properties that characterized earlier, specialized choices of the conformal action. At a deeper level, however, it is of interest to understand in detail how the gauge fields, particularly the two transla- tional fields $u^{a}$ and $v^{a}$, are interrelated. While we know beforehand that the conformal structure of spacetime is described by the Weyl curvature tensor and Weyl field strength, we want to understand how these fields arise from the fully gauged conformal group. It is gratifying to see how, in the calculations below, the symmetric and antisymmetric parts of the gauge field $v^{a}$ conspire to produce precisely the combinations of Riemannian curvatures necessary to give the two conformal fields.

Considerable theoretical effort has gone into constructing actions for and gauging the conformal group $[3,4]$. This gauging introduces four different one-forms as gauge fields. There are the usual vierbein $e^{a}$, and spin connection $\omega^{a b}$, gauge fields of the translational generators $\boldsymbol{P}_{a}$, and Lorentz generators $M_{a b}(a, b=0,1,2,3)$, respectively. In addition, the conformal algebra has a single generator of scale changes, $D$, and four generators of conformal translations, $K_{a}$. The Weyl vector $W$ is the gauge field of $D$, while gauging $K_{a}$ introduces a second vector-valued one-form $v^{a}$ similar to the vierbein, but of opposite Weyl weight.

Kaku, Townsend, and van Nieuwenhuizen [3] have shown that for a certain choice of the action functional, $v^{a}$ acts as an auxiliary field. Its field equation may be used to eliminate it from the original action. The resulting action turns out to be independent of $W$ as well. In this paper we show that the auxiliary character of $v^{a}$ is quite general, applying to all scale-invariant actions, and that the only allowed dependence on $W$ is a kinetic term.

After displaying the Lie algebra of the conformal group and the curvatures constructible from the algebra, we study the symmetries of the curvatures. This allows us to write the most general possible scale-invariant Lagrangian. Next, we vary the action with respect to $v^{a}$ and solve its field equation. This solution allows $v^{a}$ to be eliminated from the original action and results in a simple expression in terms of the two known conformally invariant tensors. Finally, we discuss what happens when the torsion two-form $T^{a}$, is allowed to be nonzero.

We begin with the Lie algebra of the generators of the conformal group. These are easiest to derive by taking 
advantage of the isomorphism between the conformal algebra and $\mathrm{O}(4,2)$, identifying $P_{a} \sim M_{a 5}+M_{a 6}$ and $K_{a} \sim M_{a 5}-M_{a 6}$ :

$$
\begin{aligned}
& {\left[M_{a b}, M_{c d}\right]=\eta_{b c} M_{a d}-\eta_{a c} M_{b d}-\eta_{b d} M_{a c}+\eta_{a d} M_{b c},} \\
& {\left[M_{a b}, P_{c}\right]=\eta_{a c} P_{b}-\eta_{b c} P_{a},} \\
& {\left[M_{a b}, K_{c}\right]=\eta_{a c} K_{b}-\eta_{b c} K_{a}, \quad\left[M_{a b}, D\right]=0} \\
& {\left[P_{a}, K_{b}\right]=2 \eta_{a b} D-2 M_{a b}, \quad\left[P_{a}, D\right]=P_{a},} \\
& {\left[P_{a}, P_{b}\right]=0, \quad\left[K_{a}, D\right]=-K_{a},} \\
& {\left[K_{a}, K_{b}\right]=0, \quad[D, D]=0 .}
\end{aligned}
$$

To demonstrate our claim, we first consider the conformal group curvature two-forms $\mathscr{R}^{A}$ constructible from the conformal connection one-form $h^{A}=d x^{\mu} h_{\mu}{ }^{A}$ $=\left(\omega^{a b}, u^{a}, v^{a}, W\right)$. These are given by

$$
\mathcal{R}^{A}=\frac{1}{2} d x^{\mu} d x^{\nu} \mathscr{R}_{\mu \nu}^{A}=d h^{A}+\frac{1}{2} h^{A} h^{B} f_{A B}{ }^{C},
$$

where the coefficients $f_{A B}{ }^{C}$ are the structure constants of the conformal algebra and the wedge product is assumed between differential forms. In terms of the component gauge fields, we find

$$
\begin{aligned}
& \mathcal{R}^{[a b]}=d \omega^{a b}+\omega^{a c} \omega_{c}{ }^{b}+2\left(u^{a} v^{b}-u^{b} v^{a}\right) \\
& \quad \equiv R^{a b}(\omega)+2\left(u^{a} v^{b}-u^{b} v^{a}\right), \\
& \mathcal{R}^{a}=d u^{a}+u^{c} \omega_{c}{ }^{a}+u^{a} W \quad\left(=T^{a} \text { when } u^{a}=e^{a}\right), \\
& \overline{\mathcal{R}}^{a}=d v^{a}+v^{c} \omega_{c}^{a}-v^{a} W, \\
& \mathcal{R}^{0}=d W+2 v^{a} u_{a} .
\end{aligned}
$$

The components of $\mathcal{R}^{a b}$, the conformal generalization of the Riemannian curvature, do not share the usual symmetries associated with $R^{a b}(\omega)$. Instead, they differ by terms depending on the antisymmetric components of $v^{a}$ :

$$
\begin{gathered}
\mathcal{R}_{\alpha \beta \mu v}=\mathcal{R}_{\mu \nu \alpha \beta}+4\left(g_{\alpha \mu} v_{[\beta v]}-g_{\alpha v} v_{[\beta \mu]}-g_{[\beta \mu]} v_{[\alpha v]}\right. \\
\left.+g_{\beta v} v_{[\alpha \mu]}\right) \\
\mathcal{R}_{\alpha[\beta \mu v]}=\frac{2}{3}\left(g_{\alpha \mu} v_{[\beta \nu]}+g_{\alpha \beta} v_{[v \mu]}+g_{\alpha v} v_{[\mu \beta]}\right) .
\end{gathered}
$$

As usual

$$
\mathcal{R}_{\alpha \beta \mu \nu}=-\mathcal{R}_{\alpha \beta \nu \mu}=-\mathcal{R}_{\beta \alpha \mu \nu},
$$

but the Ricci tensor has an antisymmetric part

$$
\mathcal{R}_{[\alpha \beta]}=-4 v_{[\alpha \beta]} .
$$

In these expressions, the gauge field $u_{\mu}{ }^{a}$ has been identified with the vierbein $e_{\mu}{ }^{a} \cdot u_{\mu}{ }^{a}$ and its inverse $u_{a}{ }^{\mu}$ are used freely to interchange tangent space and fiber indices, $(\mu, v, \ldots) \leftrightarrow(a, b, \ldots)$. The metric $g_{\mu \nu}$ is defined by $g_{\mu \nu}=e_{\mu}{ }^{a} e_{v}{ }^{b} \eta_{a b}$, where $\eta_{a b}$ is the Minkowski metric.

We now find the most general torsion-free scaleinvariant action that can be built from the curvatures (3)-(6).

The action $S$ must have zero Weyl weight, where the weight of an operator $A$ is a fixed number $w(A)$, defined whenever $A$ satisfies a commutation relation of the form

$$
[A, D]=w(A) A \text {. }
$$

The gauge field of $A$ will have the same weight as $A$. The Lagrangian density may be any zero-weight expression built from the curvature components (Weyl weights in parentheses)

$$
\mathcal{R}_{\mu \nu \alpha \beta}(+2), \quad \overline{\mathcal{R}}_{\mu \alpha \beta}(0), \quad \mathscr{R}_{\mu v}^{0}(0),
$$

together with the volume element $\sqrt{-g}(+4)$ and the tensors

$$
g_{\mu v}(+2), \quad g^{\mu v}(-2), \quad e_{\mu v \alpha \beta}(+4) .
$$

Because we have identified $u_{\mu}{ }^{a}$ with the vierbien $e_{\mu}{ }^{a}$, the remaining curvature $\mathscr{R}^{a}$ is the torsion and vanishes by assumption. Noting that only $g^{\mu v}(-2)$ can be used to eliminate the free covariant indices on the other fields, we see that every lowered index effectively changes the Weyl weight by -1 by the time it is contracted, in addition to the weights shown above. Thus, after contraction, the presence of either $\mathcal{R}_{\mu v \alpha \beta}$ or $R_{\mu \nu}^{0}$ will lower the Weyl weight by 2 from the original value of +4 due to the volume element, while the presence of $\overline{\mathcal{R}}_{\mu \alpha \beta}$ will contribute -3 . It follows that all zero-weight scalars must have exactly two factors of $\mathcal{R}_{\mu v \alpha \beta}$ or $\mathscr{R}_{\mu \nu}^{0}$ or one of each. $\overline{\mathcal{R}}_{\mu \alpha \beta}$ cannot appear if the torsion vanishes.

The Levi-Civita tensor $e_{\mu v \alpha \beta}$ can be included as needed since by the time it is fully contracted it will contribute nothing to the Weyl weight. However, since even numbers of Levi-Civita tensors can be written in terms of the metric, we need only consider those expression with either 0 or 1 factor of $e_{\mu v \alpha \beta}$.

Now, because of the lack of the usual Riemannian symmetries there are four ways to contract two factors of $R^{\alpha \beta \mu \nu}$ with the metric and seven ways to contract two factors of $\mathcal{R}^{\alpha \beta \mu v}$ with $e_{\mu v \alpha \beta}$. But since the difference from the usual symmetry depends only on the metric and $v_{[\alpha \beta]}$ as in Eqs. (7) and (9), in every case the scalars can be rearranged to one of two standard forms, plus a term quadratic in $v_{[\alpha \beta]}$. For example, using Eq. (7),

$$
\mathcal{R}_{\mu \nu \alpha \beta} \mathcal{R}^{\mu \alpha v \beta}=\frac{1}{2} \mathcal{R}_{\mu \nu \alpha \beta} \mathcal{R}^{\mu \nu \alpha \beta}+8 v_{[\alpha \beta]} v^{[\alpha \beta]} .
$$

Similarly, the pseudoscalars acquire an extra term proportional to $v_{[\alpha \beta]} v_{[\mu v]} e^{\alpha \beta \mu \nu}$. Moreover, the resulting quadratic terms in $v_{[\alpha \beta]}$ can be added in with arbitrary coefficients because these terms are proportional to the antisymmetric part of the Ricci curvature [Eq. (9)] and, hence, gauge invariant.

Certain terms are topological densities. It is interesting to note that, nonetheless, a term such as $R_{\alpha \beta \mu \nu} R_{\rho \sigma}^{\alpha \beta} e^{\mu v \rho \sigma}$ reduces to a linear combination of the purely conformal expressions [see Eqs. (17) and (19) below] $C_{\alpha \beta \mu \nu} C_{\rho \sigma}^{\alpha \beta} e^{\mu \nu \rho \sigma}$ and $C_{\mu \nu} C_{\alpha \beta} e^{\mu \nu \alpha \beta}$ with the use of the field equations.

A careful check of all possible combinations shows that the most general four-dimensional torsion-free, scaleinvariant action these curvatures allow can be written in the form 


$$
\begin{gathered}
S=\int d^{4} x \sqrt{-g}\left(a_{1} \mathcal{R}^{2}+a_{2} \mathcal{R}_{\alpha \beta} \mathcal{R}^{\alpha \beta}+a_{3} \mathcal{R}_{\alpha \beta \mu \nu} \mathcal{R}^{\alpha \beta \mu \nu}+a_{4} \mathcal{R}_{\mu \nu}^{0} \mathcal{R}^{0 \mu v}+a_{5} \mathcal{R}_{\mu \nu} \mathcal{R}^{0 \mu v}+a_{6} v_{[\mu \nu]} v^{[\mu \nu]}\right. \\
\left.+b_{1} \mathcal{R}_{\mu \nu}^{0} \mathcal{R}_{\alpha \beta}^{0} e^{\mu v \alpha \beta}+b_{2} \mathcal{R}_{\mu \nu}^{0} v_{[\alpha \beta]} e^{\mu v \alpha \beta}+b_{3} v_{[\mu \nu]} v_{[\alpha \beta]} e^{\mu v \alpha \beta}\right)
\end{gathered}
$$

All other possible terms reduce to a combination of these or fail to have the proper Weyl weight.

We have not yet considered the terms which contain the covariant derivative $D_{\mu}$ because these require special comment. The four possible even-parity derivative terms of vanishing conformal weight are

$$
\begin{aligned}
& \sqrt{-g} g^{\mu \alpha} g^{\nu \beta} D_{\nu} \overline{\mathcal{R}}_{\mu \alpha \beta}, \quad \sqrt{-g} g^{\mu \alpha} g^{\nu \beta} D_{\mu} D_{v} \mathcal{R}_{\alpha \beta}, \\
& \sqrt{-g} g^{\mu \alpha} g^{\nu \beta} D_{\alpha} D_{\beta} \mathcal{R}_{\mu \nu}^{0}, \quad \sqrt{-g} g^{\mu \nu} D_{\mu} D_{\nu} \mathcal{R},
\end{aligned}
$$

while the odd-parity counterparts of the first three are

$$
\begin{aligned}
& \sqrt{-g} e^{\mu \nu \alpha \beta} D_{v} \bar{R}_{\mu \alpha \beta}, \quad \sqrt{-g} e^{\mu v \alpha \beta} D_{\mu} D_{\nu} \mathcal{R}_{\alpha \beta}, \\
& \sqrt{-g} e^{\mu \nu \alpha \beta} D_{\alpha} D_{\beta} \mathcal{R}_{\mu \nu}^{0} .
\end{aligned}
$$

The antisymmetric terms all vanish by the Bianchi identities, but for the even ones it is necessary to generalize the divergence theorem. If we require the covariant derivative of the metric to vanish, then in addition to the usual Christoffel connection there are terms depending on the Weyl vector:

$$
\Gamma_{\mu v}^{\alpha}=\left\{\begin{array}{c}
\alpha \\
\mu v
\end{array}\right\}+\left(\delta^{\alpha}{ }_{\mu} W_{v}+\delta^{\alpha}{ }_{v} W_{\mu}-g_{\mu v} W^{\alpha}\right),
$$

with trace

$$
\Gamma_{\mu \alpha}^{\alpha}=\left\{\begin{array}{c}
\alpha \\
\mu \alpha
\end{array}\right\}+4 W_{\mu}=\partial_{\mu} \ln \sqrt{-g}+4 W_{\mu} .
$$

Therefore, the integral of the covariant divergence of a four-vector is given by

$$
\begin{aligned}
\int d^{4} x \sqrt{-g} D_{\mu} J^{\mu} & =\int d^{4} x \sqrt{-g}\left[\partial_{\mu} J^{\mu}+J^{\mu} \Gamma_{\mu \alpha}^{\alpha}+w(J) J^{\mu} W_{\mu}\right] \\
& =\int d^{4} x \sqrt{-g}\left\{\partial_{\mu} J^{\mu}+J^{\mu} \partial_{\mu} \ln \sqrt{-g}+[4+w(J)] J^{\mu} W_{\mu}\right\} \\
& =\int d^{4} x\left\{\partial_{\mu}\left(\sqrt{-g} J^{\mu}\right)+[4+w(J)] J^{\mu} W_{\mu}\right\},
\end{aligned}
$$

where $w(J)$ is the Weyl weight of the current vector. This is a surface term if and only if the current has Weyl weight $w(J)=-4$. This is precisely the weight of each of the "currents" in Eq. (15), since each is constructed to have vanishing total weight and the weight of the volume element is +4 . Therefore, each of these terms integrates to a surface term and can contribute only topologically. Note that the presence of $D_{\mu}$ precludes having any terms with more than a single curvature, so that every term is a covariant divergence.

Before moving to our main result, we recall that from the components $R_{\mu \nu}^{\alpha \beta}$ of the Riemann curvature $R^{a b}(\omega)$, and from the Weyl vector $W_{\mu}$ we can construct two conformally invariant tensors. The trace-free part of $R^{\alpha \beta}{ }_{\mu v}$ gives the Weyl curvature

$$
\begin{aligned}
C_{\mu \nu}^{\alpha \beta}= & R_{\mu \nu}^{\alpha \beta}-\frac{1}{2}\left(R_{\mu}^{\alpha}{ }_{\mu} \delta_{\nu}^{\beta}-R^{\alpha}{ }_{\nu} \delta_{\mu}^{\beta}-R_{\mu}^{\beta} \delta^{\alpha}{ }_{\nu}+R_{\nu}^{\beta}{ }_{\nu}{ }^{\alpha}{ }_{\mu}\right) \\
& +\frac{1}{6} R\left(\delta^{\alpha}{ }_{\mu} \delta^{\beta}{ }_{\nu}-\delta^{\alpha}{ }_{\nu} \delta^{\beta}{ }_{\mu}\right),
\end{aligned}
$$

while the field strength of the Weyl vector is

$$
C_{\mu v}=W_{\mu, v}-W_{v, \mu} \text {. }
$$

We are now prepared to prove the following.

Theorem 1. Every torsion-free, scale-invariant action constructible from the curvatures (3)-(6) and the metric reduces to a linear combination of the squares of the two conformal tensors $C_{\alpha \beta \mu \nu}$ and $C_{\alpha \beta}$ when the vierbien $e_{\mu}{ }^{a}$ is identified with the gauge field $u_{\mu}{ }^{a}$ of $P_{a}$ and $v_{\alpha \beta}=e_{\beta}{ }^{a} v_{\alpha a}$ is eliminated by its own field equation.

Proof. Varying the different curvatures with respect to $v_{\rho \sigma}$ yields

$$
\begin{gathered}
\delta \mathcal{R}_{\alpha \beta \mu \nu}=2\left(g_{\alpha \mu} \delta_{v}{ }^{\rho} \delta_{\beta}{ }^{\sigma}-g_{\alpha \nu} \delta_{\mu}{ }^{\rho} \delta_{\beta}{ }^{\sigma}-g_{\beta \mu} \delta_{v}{ }^{\rho} \delta_{\alpha}{ }^{\sigma}\right. \\
\left.+g_{\beta \nu} \delta_{\mu}{ }^{\rho} \delta_{\alpha}{ }^{\sigma}\right) \delta v_{\rho \sigma} \\
\delta \mathcal{R}_{\alpha \beta}=\left(4 \delta_{\alpha}{ }^{\rho} \delta_{\beta}{ }^{\sigma}+2 g_{\alpha \beta} g^{\rho \sigma}\right) \delta v_{\rho \sigma}, \\
\delta \mathcal{R}=12 g^{\rho \sigma} \delta v_{\rho \sigma}, \\
\delta \mathcal{R}_{\alpha \beta}^{0}=4 \delta_{[\alpha}{ }^{\rho} \delta_{\beta]}{ }^{\sigma} \delta v_{\rho \sigma} .
\end{gathered}
$$

Substituting these into the variation of $S$ with respect to $v_{\rho \sigma}$ gives

$$
\begin{aligned}
\delta S=\int d^{4} x \sqrt{-g}[ & 24 a_{1} \mathcal{R} g^{\rho \sigma}+a_{2}\left(8 \mathcal{R}^{\sigma \rho}+4 \mathcal{R} g^{\rho \sigma}\right)+16 a_{3} \mathcal{R}^{\sigma \rho}+8 a_{4} \mathcal{R}^{0 \rho \sigma}+4 a_{5}\left(\mathcal{R}^{0 \rho \sigma}+\mathcal{R}^{[\rho \sigma]}\right) \\
& \left.+2 a_{6} v^{[\rho \sigma]}+8 b_{1} \mathcal{R}_{\alpha \beta}^{0} e^{\rho \sigma \alpha \beta}+b_{2}\left(\mathcal{R}_{\alpha \beta}^{0}+4 v_{[\alpha \beta]}\right) e^{\rho \sigma \alpha \beta}+2 b_{3} v_{[\alpha \beta]} e^{\rho \sigma \alpha \beta}\right] \delta v_{\rho \sigma}
\end{aligned}
$$


The field equations for the symmetric and antisymmetric parts of $v_{\alpha \beta}$, respectively, are, therefore,

$$
\begin{aligned}
0= & \left(2 a_{2}+4 a_{3}\right) \mathcal{R}^{(\rho \sigma)}+\left(6 a_{1}+a_{2}\right) \mathcal{R} g^{\rho \sigma}, \\
0= & \left(8 a_{2}+16 a_{3}-4 a_{5}\right) \mathcal{R}^{[\sigma \rho]}+\left(8 a_{4}-4 a_{5}\right) \mathcal{R}^{0 \rho \sigma} \\
& +2 a_{6} v^{[\rho \sigma]}+\left(8 b_{1}+b_{2}\right) \mathcal{R}_{\alpha \beta}^{0} e^{\rho \sigma \alpha \beta} \\
& +\left(4 b_{2}+2 b_{3}\right) v_{[\alpha \beta]} e^{\rho \sigma \alpha \beta} .
\end{aligned}
$$

We treat the symmetric part first. Depending on the values of the coupling constants, there are four cases:

case 1: $\mathscr{R}^{(\alpha \beta)}=0$,

case 2: $R=0$ and $a_{2}+2 a_{3}=0$,

case 3: $\mathscr{R}_{(\alpha \beta)}-\frac{1}{4} \mathcal{R} g_{\alpha \beta}=0$ and $12 a_{1}+3 a_{2}+2 a_{3}=0$,

case 4: $a_{2}+2 a_{3}=0$ and $12 a_{1}+3 a_{2}+2 a_{3}=0$.

Each of these cases immediately leads to simplification of the action. For example, in case 1, we can immediately use Eq. (3) again, to find the symmetric part of $v_{\alpha \beta}$,

$$
v_{(\alpha \beta)}=-\frac{1}{4}\left(R_{(\alpha \beta)}-\frac{1}{6} R g_{\alpha \beta}\right),
$$

and this together with $\mathcal{R}^{(\alpha \beta)}=0$ reduces the symmetric part of the first three terms of the Lagrangian to

$$
\begin{aligned}
& a_{3}\left(\mathcal{R}_{\alpha \beta \mu v} \mathcal{R}^{\alpha \beta \mu v}\right)_{\mathrm{sym}} \\
& \quad=a_{3}\left(R_{\alpha \beta \mu \nu} R^{\alpha \beta \mu v}-2 R_{\alpha \beta} R^{\alpha \beta}+\frac{1}{3} R^{2}\right) \\
& \quad=a_{3} C_{\alpha \beta \mu \nu} C^{\alpha \beta \mu v} .
\end{aligned}
$$

In the remaining three cases the algebra is slightly more involved, but all yield precisely the same result: $a_{3} C_{\alpha \beta \mu \nu} C^{\alpha \beta \mu \nu}$. In each case the antisymmetric parts of the $a_{2}$ and $a_{3}$ terms contribute a term proportional to $v_{[\alpha \beta]} v^{[\alpha \beta]}$.

For the antisymmetric case, Eqs. (6) and (9) allow the field equation to be reduced to just four terms:

$$
\lambda_{1} v^{[\alpha \beta]}+\lambda_{2}{ }^{*} v^{[\alpha \beta]}+\lambda_{3} C^{\alpha \beta}+\lambda_{4}{ }^{*} C^{\alpha \beta}=0,
$$

where ${ }^{*} C^{\mu \nu} \equiv \frac{1}{2} C^{\rho \sigma} e_{\rho \sigma}{ }^{\mu \nu}$ and ${ }^{*} v^{\alpha \beta} \equiv \frac{1}{2} v^{\rho \sigma} e_{\rho \sigma}{ }^{\alpha \beta}$ and the new constants are defined by

$$
\begin{aligned}
& \lambda_{1}=2\left(16 a_{2}+32 a_{3}+16 a_{4}-16 a_{5}+a_{6}\right), \\
& \lambda_{2}=4\left(16 b_{1}+4 b_{2}+b_{3}\right), \\
& \lambda_{3}=4\left(2 a_{4}-a_{5}\right), \\
& \lambda_{4}=2\left(8 b_{1}+b_{2}\right) .
\end{aligned}
$$

Finally, eliminating the ${ }^{*} v^{[\alpha \beta]}$ term by combining Eq. (29) with its dual,

$$
v^{[\alpha \beta]}=\alpha C^{\alpha \beta}+\beta^{*} C^{\alpha \beta},
$$

where $\alpha$ and $\beta$ are given in terms of the $\lambda$ 's by

$$
\begin{aligned}
& \alpha=-\left(\lambda_{1}{ }^{2}+\lambda_{2}{ }^{2}\right)^{-1}\left(\lambda_{1} \lambda_{3}+\lambda_{2} \lambda_{4}\right), \\
& \beta=-\left(\lambda_{1}{ }^{2}+\lambda_{2}{ }^{2}\right)^{-1}\left(\lambda_{1} \lambda_{4}-\lambda_{2} \lambda_{3}\right) .
\end{aligned}
$$

The only possible consequences of choosing special values for the constants is to make $v^{[\alpha \beta]}$ self-dual or anti-selfdual, in which case it must vanish, or to eliminate it from the field equation altogether. In the latter case, $C_{\mu v}$ must also vanish and the antisymmetric part of the equation does not contribute to the final Lagrangian.

When Eq. (31) is substituted back into the action, the entire antisymmetric contribution reduces to a linear combination of $C_{\mu \nu} C^{\mu v}=-{ }^{*} C_{\mu \nu}{ }^{*} C^{\mu \nu}$ and $C_{\mu \nu}{ }^{*} C^{\mu \nu}$. The mixed term, however, is a divergence

$$
C_{\mu \nu}{ }^{*} C^{\mu \nu}=\frac{1}{2} e^{\mu \nu \alpha \beta} C_{\mu \nu} C_{\alpha \beta}=\partial_{v}\left(e^{\mu \nu \alpha \beta} W_{\mu} C_{\alpha \beta}\right),
$$

and, hence, contributes nothing locally.

Thus, for all values of the nine arbitrary constants in the original action, that action always reduces to

$$
S=\int d^{4} x \sqrt{-g}\left(a_{3} C_{\alpha \beta \mu \nu} C^{\alpha \beta \mu \nu}+\lambda C_{\alpha \beta} C^{\alpha \beta}\right) .
$$

We finish by proving a slightly different version, which does not result in such a clean expression for the final action, but which leaves the torsion unconstrained.

Theorem 2. Let the gauge fields be identified as in theorem 1. Then every scale invariant action allows the elimination of $v_{\alpha \beta}$.

Proof. If the torsion does not vanish, it is possible to write further scale-invariant terms, but they do not change the auxiliary character of $v_{\mu \nu}$. There are kinetic terms for $T_{\mu \nu \alpha}(+2)$ given by the distinct contractions of

$$
\begin{aligned}
& \sqrt{-g} D_{v} T_{\alpha \beta \mu} D_{v^{\prime}} T_{\alpha^{\prime} \beta^{\prime} \mu^{\prime}} g^{\rho \sigma} g^{\gamma \delta} g^{\rho^{\prime} \sigma^{\prime}} g^{\gamma^{\prime} \delta^{\prime}}, \\
& \sqrt{-g} D_{v} T_{\alpha \beta \mu} D_{v^{\prime}} T_{\alpha^{\prime} \beta^{\prime} \mu^{\prime}} g^{\rho \sigma} g^{\gamma \delta} e^{\rho^{\prime} \sigma^{\prime} \gamma^{\prime} \delta^{\prime}} .
\end{aligned}
$$

Also there are several possible torsion- $v_{\mu \nu}$ couplings:

$$
\begin{aligned}
& \sqrt{-g} \overline{\mathcal{R}}_{\mu \alpha \beta} T^{\mu \alpha \beta}, \quad \sqrt{-g} \overline{\mathcal{R}}_{\mu \alpha \beta} T^{\alpha \beta \mu}, \quad \sqrt{-g} \overline{\mathcal{R}}_{\alpha \beta}^{\alpha} T_{\mu}{ }^{\mu \beta} \text {, } \\
& \sqrt{-g} \bar{R}_{\rho \alpha \beta} T^{\rho}{ }_{\mu \nu} e^{\alpha \beta \mu \nu}, \quad \sqrt{-g} \overline{\mathcal{R}}_{\rho \alpha \beta} T_{\mu}{ }^{\rho}{ }_{v} e^{\alpha \beta \mu \nu}, \\
& \sqrt{-g} \overline{\mathcal{R}}_{\alpha \rho \beta} T_{\mu}{ }^{\rho}{ }_{v} e^{\alpha \beta \mu \nu}, \quad \sqrt{-g} \overline{\mathcal{R}}_{\alpha \rho \beta} T^{\rho}{ }_{\mu \nu} e^{\alpha \beta \mu \nu}, \\
& \sqrt{-g} \overline{\mathcal{R}}_{\alpha \beta \mu} T_{\rho v}^{\rho} e^{\alpha \beta \mu \nu}, \quad \sqrt{-g} \overline{\mathcal{R}}_{\rho \alpha}^{\rho} T_{\beta \mu \nu} e^{\alpha \beta \mu \nu} \text {, }
\end{aligned}
$$

and similar couplings with $D_{\alpha} \mathcal{R}_{\mu \nu}^{0}(0)$ or $D_{\alpha} \mathcal{R}_{\mu \nu}(0)$ replacing $\overline{\mathscr{R}}_{\alpha \mu \nu}$.

Arbitrary linear combinations of these terms may be added. All of the new terms contain $v_{\mu \nu}$ in some form, but in every case the dependence is linear. When varied with respect to $v_{\mu v}$ and, if necessary, integrated by parts, these linear terms will give contributions to the expression for $v_{\mu v}$ which depend on the torsion and $v$ independent curvatures and their first derivatives. Thus $v_{\mu \nu}$ may still be eliminated. Q.E.D.

The reason for this reduction is that there is no single metric structure on a general four-dimensional manifold consistent with the full symmetry. For clearly the action of $K_{a}$ and $D$ leave only the light-cone structure unchanged. Another way to see the problem is to note that the conformal group is a representation of $\mathrm{O}(4,2)$. An action constructed from four-dimensional tensors will not, in general, be invariant under a six-dimensional rotation group. $C_{\mu \nu}$ and $C_{\alpha \beta \mu \nu}$, however, may be written as a sixdimensional vector and matrix, respectively, and therefore can form conformally invariant combinations. 
In one sense the role of $v_{\mu \nu}$ in enforcing the emergence of the Weyl curvature is quite natural and satisfying. After all, $C_{\alpha \beta \mu \nu}$ and $C_{\mu \nu}$ are the conformally invariant parts of the curvature and must emerge somehow from the gauge fields. From theorem 1 it is clear that $v_{\mu \nu}$ produces this reduction, and it is even possible to see in a term-by-term way how $v_{\mu \nu}$ brings in the extra Ricci terms required to produce $C_{\alpha \beta \mu v}$.

Our ability to remove $v_{\alpha \beta}$ hinges on two factors. First, the scale invariance requires the curvatures to enter the action at most quadratically so that the variation with respect to $v_{\alpha \beta}$ leaves an equation linear in $v_{\alpha \beta}$. Second, the particular Weyl weight and index structure of $\overline{\mathcal{R}}_{\mu \alpha \beta}$ precludes the possibility of a kinetic term for $v_{\alpha \beta}$.

One way that the auxiliary character of $v_{\alpha \beta}$ can be altered is by allowing divisors in the action. Since the scalar $v^{2} \equiv v_{[\alpha \beta]} v^{[\alpha \beta]}$ is permitted, we might consider terms of the form

$$
\sqrt{-g} \overline{\mathcal{R}}_{\mu \alpha \beta} \overline{\mathcal{R}}^{\mu \alpha \beta}\left(v^{2}\right)^{-1 / 2},
$$

which has the correct weight and introduces a kinetic term. Of course, if powers of $v^{2}$ are allowed, then any scalar can be made weightless, and there is little constraint on what terms could appear. Naturally, with such terms the vanshing of $v^{2}$ could be disastrous.

Returning to the discussion at the beginning, we consider the possibility of finding additional symmetry in the gauging of the full conformal group. An additional SU(2) or SU(3) symmetry could explain the weak or strong force. From the auxiliary character of $v_{\alpha \beta}$, it appears that the symmetry reduces back to the Weyl group where we started. However, the reduction of the symmetry to a smaller group is somewhat puzzling, because in addition to the elimination of the gauge field $v_{\alpha \beta}$, we have lost the symmetry that holds between $P_{a}$ and $K_{a}$. The Lie algebra of the conformal group [Eq. (1)] displays symmetry between these two generators, but the choice $u^{a}=e^{a}$ for the vierbein throws $v_{\mu v}$ into the role of an auxiliary field. Had we chosen $v^{a}=e^{a}$ instead, $u^{a}$ could have been eliminated. In a subsequent paper [5], we will explore this $P$ $K$ symmetry in detail and take a fresh look at gauging the conformal group in an attempt to preserve this additional symmetry explicitly.
[1] H. Weyl, Sitzungsber. Preuss. Akad. Wiss. 26, 465 (1918); Math. Z. 2, 384 (1918); Ann. Phys. 59, 101 (1919); Phys. Z. 21, 649 (1920); 22, 473 (1921); Z. Phys. 56, 330 (1929).

[2] J. T. Wheeler, Phys. Rev. D 41, 431 (1990).

[3] M. Kaku, P. K. Townsend, and P. van Nieuwenhuizen, Phys. Lett. 69B, 304 (1977).

[4] P. G. O. Freund, Ann. Phys. (N.Y.) 84, 440 (1974); F.
Mansouri, Phys. Rev. Lett. 42, 1021 (1979); Phys. Rev. D 24, 1056 (1981); F. Mansouri and C. Schaer, Phys. Lett. 101B, 51 (1981); F. Mansouri, J. Math. Phys. 24, 890 (1983).

[5] J. T. Wheeler, Utah State University Report No. FTG105-USU, 1991 (unpublished). 